\title{
ANÁLISIS DEL DESPACHO ANTICIPADO Y DIFERIDO EN LA INTENDENCIA DE ADUANA MARÍTIMA DEL CALLAO 2015-2018
}

\author{
ANALYSIS OF EARLY AND DEFERRED CLEARANCE AT CALLAO MARITIME \\ CUSTOMS AGENCY 2015-2018
}

Juan Alvarez Aroni

Universidad Nacional Mayor de San Marcos Lima, Perú

ORCID: https://orcid.org/0000-0003-0802-080X

Correo electrónico: jalvarezaroni@gmail.com

\section{RESUMEN}

Objetivo: Estudiar los tiempos empleados durante el proceso de importación de mercancías mediante el despacho anticipado y diferido en la Intendencia de Aduana Marítima del Callao durante el periodo 2015 - 2018. Método: La investigación se enmarca dentro del método deductivo. Se ha empleado información recopilada sobre el tiempo de la cadena logística de importación de mercancías en los periodos 2015-2018 a través de la Intendencia de Aduana Marítima del Callao, que fue analizada usando la prueba de Kolmogorov - Smirnov, a fin de determinar su normalidad y la ecuación de predictibilidad. Resultados: Analizados los tiempos empleados en el proceso de importación en la modalidad de despacho anticipado y diferido, se verifica qué tramos de la cadena logística explican el comportamiento de esta, lo que permite crear una ecuación de predictibilidad de comportamiento. Conclusiones: La modalidad de despacho diferido tiene mejores tiempos en el proceso de importación de mercancías, por lo que la Administración Aduanera deberá de formular estrategias a fin de mejorar el despacho anticipado al ser de uso obligatorio a partir del 2020.

Palabras clave: Importación; despacho anticipado; despacho diferido; Aduana Marítima del Callao.

\begin{abstract}
Objective: Study the times used during the merchandise import process by means of anticipated and deferred clearance at the Callao Maritime Customs Office during the period 2015-2018. Method: The research is framed within the deductive method. Information collected on the time of the logistics chain of import of goods in the periods 2015 - 2018 through the Intendancy of Maritime Customs of Callao, which was analyzed using the Kolmogorov - Smirnov test, in order to determine its normality and used the predictability equation. Results: Analyzed the times used in the import process in the modality of anticipated and deferred dispatch, it is verified which sections of the logistics chain explain the behavior of this, which allows creating a behavior predictability equation. Conclusions: The deferred clearance modality has better times in the process of importing merchandise, so the Customs Administration must formulate strategies in order to improve early clearance as it is mandatory for use from 2020.
\end{abstract}

Keywords: Import; advance clearance; deferred clearance; Callao Maritime Customs.

(c) Los autores. Este artículo es publicado por la Revista Quipukamayoc, Universidad Nacional Mayor de San Marcos. Este es un artículo de acceso abierto, distribuido bajo los términos de la Licencia Creative Commons Atribución-NoComercial-Compartirlgual 4.0 Internacional.(http://creativecommons.org/licenses/by-nc-sa/4.0/), que permite el uso no comercial, distribución y reproducción en cualquier medio, siempre que la obra original sea debidamente citadas. 


\section{INTRODUCCIÓN}

No es un secreto que estamos en un mundo globalizado donde el comercio transfronterizo crece cada día más y más a un nivel exponencial. Es en este contexto que las variables, costos y tiempos, juegan un papel predominante; todos los países apuntan a reducir sus tiempos y costos de importación y exportación haciendo que sus controles sean más ágiles y eficientes.

Dentro del contexto mundial, en el indicador referido al cumplimiento fronterizo, Francia, Luxemburgo, Dinamarca, Bélgica, Croacia, Hungría, Italia, Rumania, Eslovaquia, Eslovenia, Países Bajos, República Checa, Portugal, España, Polonia y Austria, necesitan tan solo una hora para los controles tanto de exportación como de importación. En el caso peruano, el proceso de importación requiere 72 horas, además requiere 48 horas adicionales para cumplir con los requisitos documentales requeridos, por lo que en total para el proceso de despacho de importación se requiere en promedio 120 horas o un total de 5 días (Doing Business, 2019).

En el informe de Doing Business, en lo referente al comercio transfronterizo, se puede apreciar que el Perú se encuentra en la posición 102 de 188 economías analizadas a nivel mundial, y en América Latina ocupamos la posición 16 de 32 economías, estando El Salvador, Panamá, República Dominicana, México y Puerto Rico ocupando las primeras 5 posiciones. Este panorama nos da una idea de cuánto se debe mejorar como país para ser competitivos en el comercio internacional; las políticas gubernamentales deben apuntar a una óptima gestión logística en toda la cadena de importación a fin de mejorar los tiempos, lo que conllevará a una reducción de costos, que al final dará mayores ventajas para competir a nivel mundial en el intercambio comercial de productos de importación y exportación.

El Estado peruano debe apuntar a reducir los tiempos, lo que conllevará a reducir los costos en los procesos de importación, es así que junto con los operadores portuarios quien juega un papel importante para lograr este objetivo es la Superintendencia Nacional de Aduanas y Administración Tributaria (SUNAT). Por ello, ante el problema de los elevados tiempos de atención de los procesos de importación, ha diseñado diversas estrategias para tratar de reducir estos tiempos de atención, siendo una de ellas, sino la más importante, incentivar el uso o acogimiento por parte de los importadores al despacho anticipado, que fue recogido y mejorado dentro de la Ley General de Aduanas aprobado por Decreto Legislativo 1053 el 27 de junio del 2008.

Dentro de los últimos siete años, la SUNAT ha centrado sus esfuerzos en tratar de que los importadores utilicen la modalidad de despacho anticipado a fin de mejorar los tiempos y reducir los costos, ofreciendo como principal ventaja, en comparación con la modalidad de despacho diferido, la opción de que el importador pueda solicitar la destinación de sus mercancías importadas mediante la numeración de la Declaración de Importación de Mercancías (DAM) y obtener el levante autorizado antes de la llegada del medio de transporte (USAID, 2012). Con el despacho anticipado se pretende eliminar de la cadena logística el proceso mediante el cual las mercancías ingresen a un depósito temporal pudiendo el importador retirar sus mercancías directamente del terminal portuario una vez terminada la descarga de la nave, ahorrando un tiempo significativo en la disposición de sus mercancías. Por ello, la SUNAT pretende que los tiempos de liberación de mercancías se reduzcan en un futuro de 132 a 15 horas (PQS, 2018). Sin embargo, pese a las ventajas que ofrece el despacho anticipado, el acogimiento a esta modalidad no habría tenido el resultado esperado.

El problema es que los importadores no consideran al despacho anticipado como una opción para reducir sus costos ni tiempos de atención; en este sentido Cosio (2017) afirma: "Debe tenerse en cuenta que a la fecha pese a todos los incentivos legales recibidos el sistema anticipado no ha alcanzado un uso superior al $38 \%$ del total de despachos" (p. 240).

Sin embargo, en las modificaciones a la Ley General de Aduanas (Decreto Legislativo No 1433) el Estado Peruano establece que la modalidad del despacho anticipado es obligatoria para todos los regímenes salvo las excepciones que seguirán como despacho diferido. Esta obligatoriedad obligará a los importadores a asumir costos y tiempos que la administración no lo mide en el cálculo de sus tiempos, pero que necesariamente lo tiene que realizar para cumplir con la ley.

Teniendo en cuenta el nivel de acogimiento del despacho anticipado podemos ver que el despacho diferido viene siendo aún el más utilizado; ante esta situación desde la entrada en vigor del Decreto Legislativo No 1433, en el cual se establece la obligatoriedad del despacho anticipado, a partir del 31 de diciembre del 2019 se ha iniciado la discusión si esta obligatoriedad es viable, factible o no.

Con esta investigación ha quedado demostrado que no es viable la obligatoriedad del despacho anticipado en nuestro país, toda vez que el despacho diferido es el preferido por la mayoría de los importadores en nuestro país, ya que permite tramitar el despacho con la mercancía en un depósito temporal sin la necesidad de la obligatoriedad de presentar la documentación antes de la llegada de la nave. (Estrada, 2018, p. 101) 
Si la Administración Aduanera busca incrementar los niveles de acogimiento al despacho anticipado es necesario que ofrezca mayores beneficios a los importadores y no que imponga una modalidad mediante un mandato legal, esto equivaldría a decirle al importador: si no entiendes que es beneficioso el despacho anticipado a las buenas, lo entenderás a las malas (Guadalupe, 2019).

Una de las principales ventajas del despacho anticipado en comparación con el diferido, con la que se ha tratado que mayores importadores se acojan a esta modalidad, es la reducción de los tiempos en el proceso logístico de importación, lo que finalmente se verá traducido en un ahorro de dinero. Por ello, en los últimos años la SUNAT ha venido utilizando herramientas virtuales y nuevas tecnologías de la información con el propósito de mejorar la trazabilidad de los despachos de importación. Es en ese sentido que desde julio del 2018 se implementó la herramienta virtual denominada "tiempo de importación", que permite al usuario conocer en el momento los detalles del proceso de nacionalización de sus mercancías, comparando los tiempos de atención del despacho anticipado y diferido (Andina, 2018).

También se suma la implementación del sistema de notificaciones electrónicas al buzón del usuario, que indica al importador que su carga ya llegó al país. Junto con ello, en el 2019, se viene desarrollando el programa FAST (Facilitación Aduanera, Seguridad y Transparencia) que consiste en la implementación de diversos cambios tecnológicos en todas las modalidades de despacho, locaciones y regímenes aduaneros que se desarrollarán en una misma plataforma y no por separado como actualmente sucede (APAM, 2018).

\section{MATERIALES Y MÉTODOS}

La investigación se enmarca dentro del método deductivo puesto que se analizó toda la cadena logística en las dos modalidades de despacho para su posterior análisis desagregado a nivel de los tramos que componen ambas cadenas logísticas de importación, lo que finalmente permitió buscar la relación que existente entre los tramos que componen la cadena logística y que puedan estar alterando los tiempos de atención en ambas modalidades de despacho (anticipado y diferido); por consiguiente, esta investigación fue de tipo descriptivo correlacional.

Mejorar los tiempos de atención desde que se estableció el despacho anticipado, hace ya más de 10 años, es una tarea y realidad compleja; por ello, para poder verificar y determinar si estos tiempos a lo largo de los años ha ido mejorando, se ha tomado como período de análisis los tiempos de atención de las importaciones realizadas bajo la modalidad de despacho tanto anticipado como diferido entre los años 2015 y 2018. Se utilizó la prueba de Kolmogorov - Smirnov (K-S) a fin de determinar si estos datos de tiempos de atención corresponden a una distribución normal. Al respecto, Romero (2016) refiere que "las pruebas de bondad de ajuste se utilizan para contrastar si los datos de la muestra pueden considerarse que proceden de una determinada distribución o modelo de probabilidad" (p. 105). Una vez determinada la normalidad de los datos, se infirió una ecuación que podría explicar o predecir el comportamiento de los tiempos de atención a futuro y la relación que puedan tener los tramos que componen la cadena logística de importación.

Para el análisis de los tiempos se establecieron tramos con sus respectivos hitos para ambas modalidades de despacho de importación (anticipado y diferido). Estos tramos pueden servir para establecer acciones a desarrollar por la Administración Aduanera y mejorar lo tiempos de atención. Para los propósitos de la investigación se utilizó el software Minitab 19, que es muy usado para realizar análisis de datos estadísticos y mejora de procesos.

\section{RESULTADOS}

Las cadenas logísticas del despacho anticipado y diferido tienen sus particularidades que las hacen diferentes. El despacho anticipado podemos dividirlo en 5 tramos, mientras que el despacho diferido en 7 , desde que se embarcan las mercancías hasta que son retiradas del terminal portuario o depósito temporal, como se muestra a continuación:

\section{Despacho anticipado}

Tramo 1: Desde el embarque de las mercancías hasta la numeración de la DAM.

Tramo 2: Desde la numeración de la DAM hasta el levante autorizado.

Tramo 3: Desde el levante autorizado hasta la llegada de la nave.

Tramo 4: Desde la llegada de la nave hasta el término de la descarga.

Tramo 5: Desde el término de la descarga hasta el retiro de las mercancías del terminal portuario.

\section{Despacho diferido}

Tramo 1: Desde el embarque de las mercancías hasta la llegada.

Tramo 2: Desde la llegada hasta el término de la descarga.

Tramo 3: Desde el término de la descarga hasta el ingreso de las mercancías al depósito temporal. 
Tramo 4: Desde el ingreso de las mercancías al depósito temporal hasta la numeración de la DAM.

Tramo 5: Desde la numeración de la DAM hasta el pago de tributos.

Tramo 6: Desde el pago de tributos hasta el levante autorizado.

Tramo 7: Desde el levante autorizado hasta el retiro del depósito temporal.

A continuación, se evalúa el despacho anticipado desde el momento del embarque de las mercancías en el país de origen, hasta el momento que el importador retira las mercancías del depósito temporal o punto de llegada. En la tabla 1, se puede apreciar los tres tipos de canales de control y la cantidad de DAM atendidas por la Administración Aduanera junto a los tiempos que se emplearon en cada tramo.

Se evaluó un total de 456614 DAM anticipadas correspondientes al período de análisis 2015 - 2018; de ellas 11 547 tienen como canal de control naranja; 6 610, canal rojo; y 438 457, canal verde.

Como se aprecia, el 96\% de las DAM fueron asignadas a canal verde, asimismo se observa que, de los aproximados 55 días que transcurren desde el embarque de las mercancías hasta que se retiran del puerto, casi 48 días corresponden al transporte internacional, es decir, antes que el agente de aduana numere una DAM (Tramo 1). El agente de aduana numera la DAM en un aproximado de menos de cinco días antes de la llegada del medio de transporte, si bien se obtiene el levante autorizado incluso antes de la llegada de la nave (Tramo 2 y 3), el retiro de la mercancía se realiza en un promedio de 3 días posteriores a la llegada del medio de transporte (Tramo 4 y 5 ).

De acuerdo a lo indicado anteriormente, se puede decir que, en el primer tramo no se puede tener influencia para mejorarlo, porque el tiempo del transporte internacional es un factor externo en el cual poco puede influir la
SUNAT, puesto que la travesía depende de la distancia geográfica que recorren los buques portacontenedores; sin embargo, podemos proponer que el agente de aduana numere su DAM cercana a la fecha de llegada de la nave y hacer que este operador tenga un tiempo mínimo dedicado a los trámites aduaneros.

De otro lado, se aprecia que desde la llegada de la nave hasta el retiro de la mercancía del terminal portuario transcurren aproximadamente 3 días (Tramo 4 y 5), incluso contándose con levante autorizado para disponer de sus mercancías; por consiguiente, el importador tiene que estar pendiente del momento del retiro de la mercancía, que no ocurre inmediatamente, por lo que sería necesario analizar las razones de la demora en el retiro de las mercancías.

De acuerdo con el comportamiento de los datos analizados se colige que los tramos 3 y 5 son los que ameritarían un mayor análisis. En el tramo del levante, a la llegada del medio de transporte (Tramo 3), se observa que se tiene valores negativos, lo que significa que el levante es posterior a la llegada del medio de transporte; sin embargo, en el canal verde se aprecia que se demoran casi un día para obtener el levante (0,749 días) posteriores a la llegada, en el canal rojo aproximadamente 7 días $(6,258$ días) y en el canal naranja una media de más de dos días (2,779 días). Teniendo en cuenta esta situación, donde se aprecia que existen levantes posteriores a la fecha de llegada, analizando las DAM se puede concluir que esto sucede porque la numeración de los anticipados puede ocurrir en la misma fecha de la llegada, incluso en horas posteriores a la llegada, ya que el sistema de SUNAT solo valida fechas más no horas. Por otro lado, la mayor dispersión del tiempo se tiene en el canal rojo, donde el reconocimiento físico hace que el levante se demore mucho más que el tiempo que se podría demorar el promedio del tiempo en despacho diferido.

En el caso del Tramo 5 (Descarga - Retiro Terminal Portuario), lo más resaltante que se evidencia es que en general los importadores demoran casi tres días (entre 2,545 y

Tabla 1

Tiempos Promedio Despacho Anticipado (Días)

\begin{tabular}{lccccccccccccc}
\hline \multirow{2}{*}{ CANAL } & \multicolumn{2}{c}{ DAM } & \multicolumn{4}{c}{ TRAMO 1 } & \multicolumn{2}{c}{ TRAMO 2 } & \multicolumn{2}{c}{ TRAMO 3 } & \multicolumn{2}{c}{ TRAMO 4 } & \multicolumn{2}{c}{ TRAMO 5 } & TIEMPO \\
& Cantidad & $\%$ & Tiempo & $\%$ & Tiempo & $\%$ & Tiempo & $\%$ & Tiempo & $\%$ & Tiempo & $\%$ & TOTAL \\
\hline Verde & 438457 & $96 \%$ & 47,4 & $67 \%$ & 4,35 & $6 \%$ & $-0,74$ & $-1 \%$ & 1,10 & $2 \%$ & 2,54 & $4 \%$ & 54,65 \\
Naranja & 11547 & $3 \%$ & 63,7 & $90 \%$ & 5,78 & $8 \%$ & $-2,77$ & $-4 \%$ & 1,16 & $2 \%$ & 2,75 & $4 \%$ & 70,62 \\
Rojo & 6610 & $1 \%$ & 57 & $81 \%$ & 9,24 & $13 \%$ & $-6,25$ & $-9 \%$ & 0,98 & $2 \%$ & 2,77 & $4 \%$ & 63,73 \\
TOTAL & 456614 & $100 \%$ & & & & & & & & & & & \\
\hline
\end{tabular}

Elaborado en base a la información proporcionada por la Superintendencia Nacional de Aduanas y Administración Tributaria - SUNAT 
2,772 días) en retirar sus mercancías una vez que se ha terminado la descarga, siendo lo más llamativo lo que ocurre con las DAM con canal de control verde, que no requieren ningún tipo de revisión y obtienen el levante automático una vez cancelados los tributos, y a pesar de ello demoran en ser retirados, tiempos incluso mayores que las DAM con canal de control rojo y naranja que tienen procesos de control después de la llegada.

En la figura 1, el gráfico de cajas muestra el comportamiento que tienen las DAM analizadas con respecto a la mediana en los tramos 3 y 5 .

Según el análisis de normalidad de la información, esta responde a una distribución normal; es así como se aprecia que tanto el tramo 3 y el tramo 5, según los canales de control $($ Naranja $=$ D, Rojo $=$ F y Verde $=$ V) muestran un comportamiento típico de una distribución normal (el valor de p es mayor de 0,05$)$. Por consiguiente, se concluye que los datos analizados tienen un carácter de generalidad. La figura 2, muestra el comportamiento de la data en los seis casos con la correspondiente prueba de normalidad.

Por tanto, en función a los tramos 3 y 5, se puede llegar a la conclusión que, efectuando un análisis de correlación, tal como se ve en la figura 2, la ecuación de predictibilidad del tiempo a demorarse en el despacho anticipado sería:

$$
\text { TTotal }=52,945+1,398 \mathrm{~T} 3+1,178 \mathrm{~T} 5
$$

Donde:

TTotal $=$ Tiempo total de la cadena logística del despacho anticipado.

T3 = Tramo 3 del despacho anticipado.

T5 = Tramo 5 del despacho anticipado.

Esta ecuación indica que, mientras se tiene un tiempo constante de 52,945 horas, el despacho anticipado variará teniendo en cuenta el comportamiento de los tramos 3 y 5 ; es decir, mientras mayor sean estos valores, mayor será el tiempo total de despacho anticipado.

Con respecto a despacho diferido, se generó el estudio de las medias de tiempo que resulta a cada tramo, tal como podemos apreciar en la tabla 2.

Para el análisis se tomaron en cuenta 1338022 declaraciones, de las cuales las declaraciones con canal de control verde representan el $85 \%$, con canal naranja el $8 \%$ y con

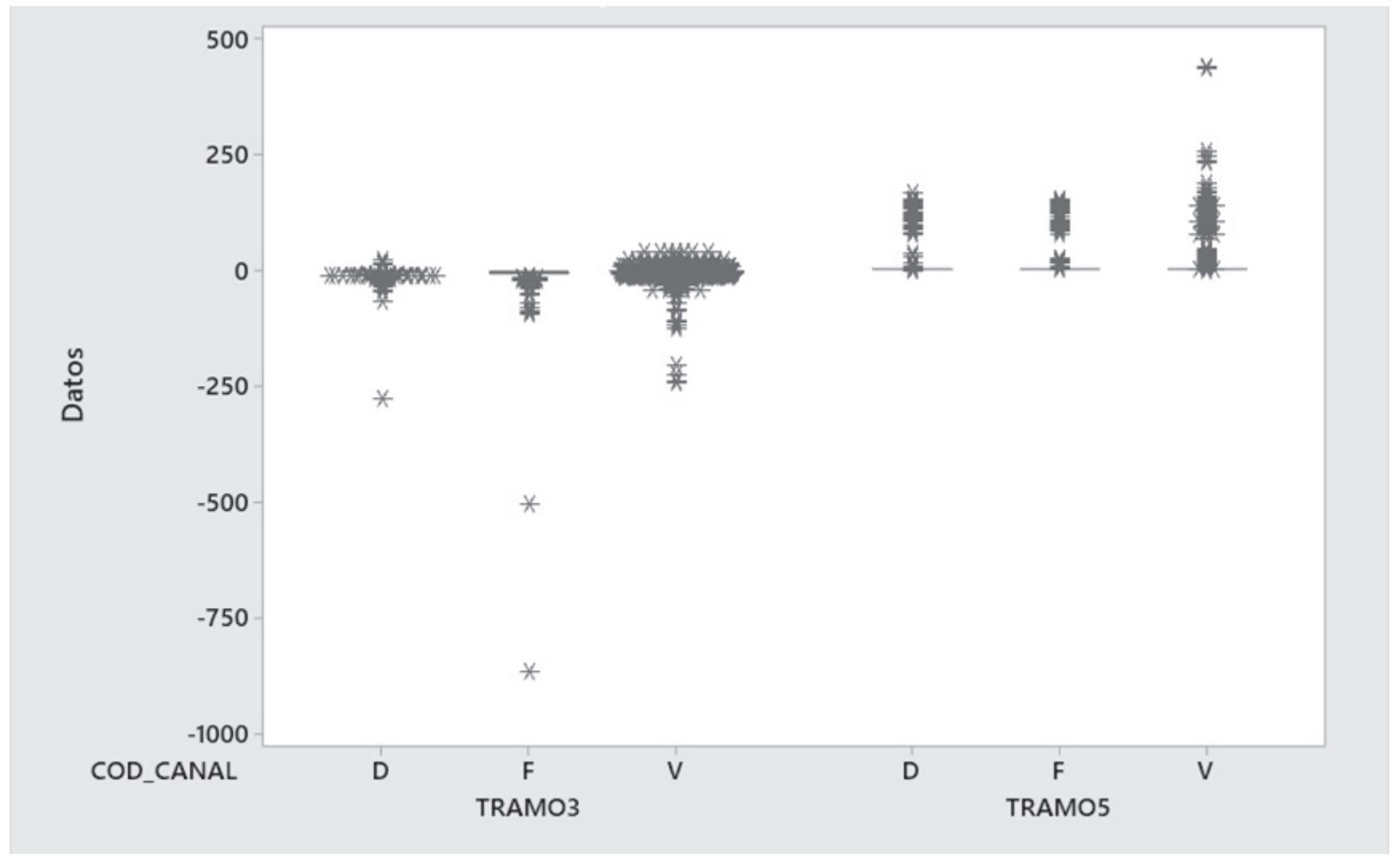

Figura 1. Gráfico de dispersión de DAM de los tramos 3 y 5 respecto a la mediana. Los asteriscos representan a las DAM y su comportamiento por canal de control ( $\mathrm{V}=$ verde, $\mathrm{D}=$ naranja y $\mathrm{F}=$ rojo) dentro de los tramos analizados. Elaborado en base a la información proporcionada por la SUNAT. 


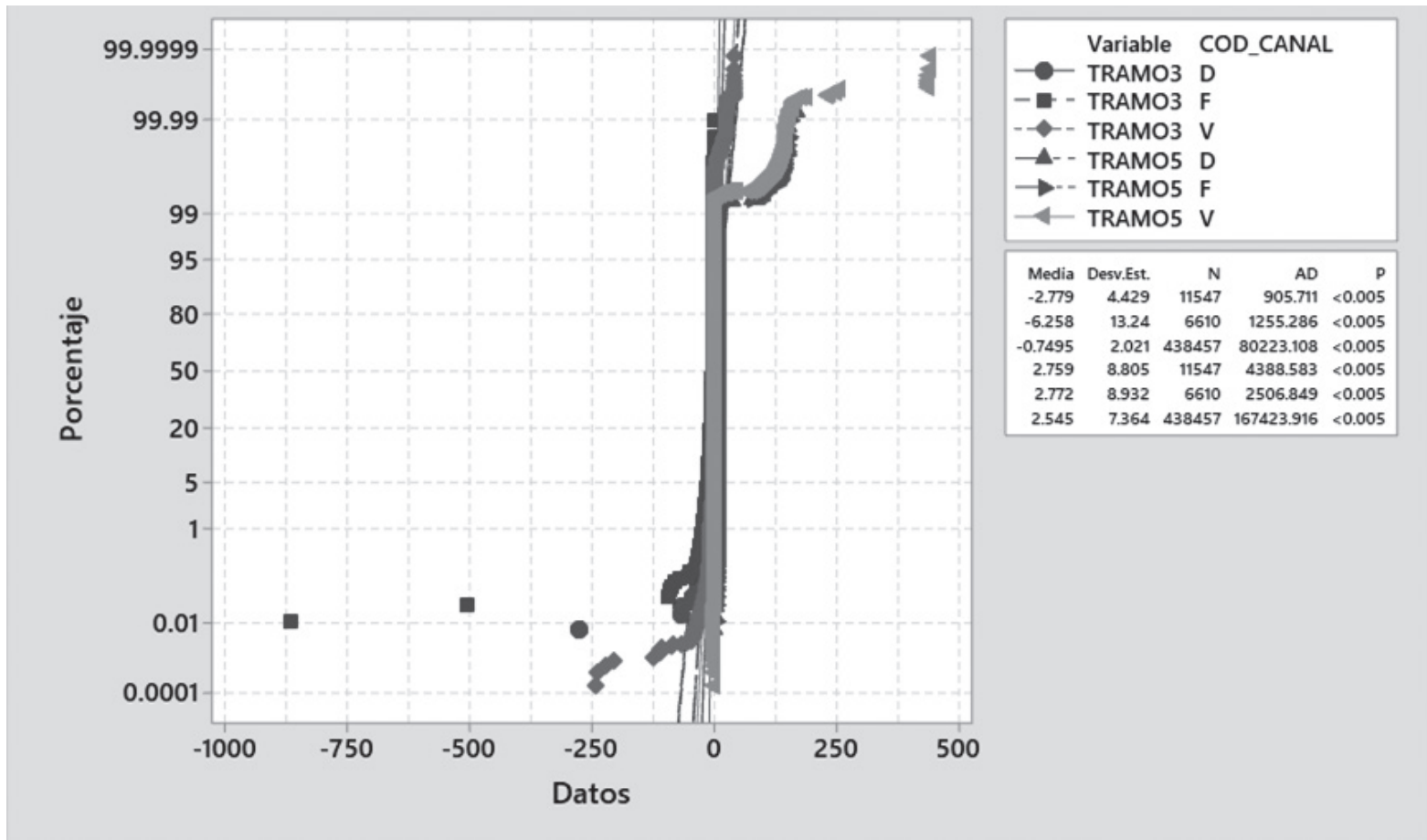

Figura 2. Prueba de normalidad Tramo 3 y Tramo 5 (Normal - 95\% de IC). Elaborado en base a la información proporcionada por la SUNAT.

Tabla 2

Tiempos Promedio Despacho Diferido (Días)

\begin{tabular}{|c|c|c|c|c|c|c|c|c|c|c|c|c|c|c|c|c|c|}
\hline \multirow{2}{*}{ CANAL } & \multicolumn{2}{|c|}{ DAM } & \multicolumn{2}{|c|}{ TRAMO 1} & \multicolumn{2}{|c|}{ TRAMO 2} & \multicolumn{2}{|c|}{ TRAMO 3} & \multicolumn{2}{|c|}{ TRAMO 4} & \multicolumn{2}{|c|}{ TRAMO 5} & \multicolumn{2}{|c|}{ TRAMO 6} & \multicolumn{2}{|c|}{ TRAMO 7} & \multirow{2}{*}{$\begin{array}{c}\text { TIEMPO } \\
\text { TOTAL }\end{array}$} \\
\hline & Cantidad & $\%$ & Tiempo & $\%$ & Tiempo & $\%$ & Tiempo & $\%$ & Tiempo & $\%$ & Tiempo & $\%$ & Tiempo & $\%$ & Tiempo & $\%$ & \\
\hline VERDE & 1139362 & $85 \%$ & 30 & $72 \%$ & 1 & $2 \%$ & 0,93 & $2 \%$ & 3,8 & $9 \%$ & 0,56 & $1 \%$ & 0,08 & $0 \%$ & 4,87 & $12 \%$ & 40,92 \\
\hline NARANJA & 101524 & $8 \%$ & 30 & $66 \%$ & 0,98 & $2 \%$ & 1,25 & $3 \%$ & 6,29 & $14 \%$ & 1,03 & $2 \%$ & 3,13 & $7 \%$ & 2,32 & $5 \%$ & 44,66 \\
\hline ROJO & 97136 & $7 \%$ & 30 & $60 \%$ & 0,97 & $2 \%$ & 1,23 & $3 \%$ & 6,87 & $14 \%$ & 1,12 & $2 \%$ & 7,01 & $14 \%$ & 2,19 & $4 \%$ & 49,05 \\
\hline TOTAL & 1338022 & $100 \%$ & & & & & & & & & & & & & & & \\
\hline
\end{tabular}

Elaborado en base a la información proporcionada por la Superintendencia Nacional de Aduanas y Administración Tributaria - SUNAT

canal rojo el 7\%. Asimismo, el tiempo total de liberación de una DAM canal verde es aproximadamente de 41 días (40,92 días), de los cuales 30 días corresponden al tiempo transcurrido entre el embarque y la llegada del medio de transporte (tramo 1); al igual que en despacho anticipado, este tiempo corresponde a la distancia geográfica que recorren los buques portacontenedores antes de llegar al puerto del Callao, sobre este no se tiene injerencia para poder plantear alternativas de mejora. Por otro lado, un promedio aproximado de casi 4 días (3,8 días) corresponde al tramo 4 que comprende al tiempo transcurrido desde el ingreso de las mercancías al Depósito Temporal hasta la numeración de la DAM efectuada por el Agente de Aduana, y finalmente aproximadamente 5 días $(4,87$ días) es el tiempo que transcurre desde que la autoridad aduanera otorga el levante a las mercancías hasta el retiro de estas del Depósito Temporal.

De lo indicado anteriormente y de la información que se analizó, se observa que los tramos a analizar son el tramo 4 y el tramo 7 , que es el tiempo que transcurre desde el momento en que la mercancía ingresa al almacén hasta que es destinada al régimen de importación para el consumo. El otro tramo para analizar es desde que la mercancía tuvo el levante hasta que el dueño o consignatario retira la mercancía del depósito temporal. 
El grafico de cajas en la figura 3 muestra el comportamiento que tienen las DAM analizadas con respecto a la mediana en los tramos 4 y 7.

Del análisis de la información, se observa que desde que la mercancía ingresa al almacén hasta que es destinada al régimen de importación, es el canal verde el que presenta mayor tiempo para su destinación, puesto que las DAM con canal de control naranja y rojo son numeradas en un tiempo muy cercano al ingreso. De otro lado, se tiene que las mercancías contenidas en las DAM con canal de control verde son las que demoran más en ser retiradas del depósito temporal.

Luego de analizar la normalidad de la información con la que se trabajó para el despacho diferido, esta nos muestra que responde a una distribución normal, como podemos ver que en el tramo 4 y en el tramo 7 , según los canales de control, la data de los seis casos estudiados muestra un comportamiento típico de una distribución normal (el valor de p es mayor de 0,05). Esto nos indica, al igual que en el despacho anticipado, que los tiempos analizados tienen un carácter de generalidad. La figura 4, muestra el comportamiento de la data en los seis casos con la correspondiente prueba de normalidad.

Considerando el análisis de regresión de los tramos 4 y 7 con respecto al total del tiempo tal como se ve en la figura
4, la ecuación de predictibilidad del tiempo a demorarse en el despacho diferido sería:

$$
\text { TTotal }=793,095+1,03733 \mathrm{~T} 4+0,976965 \mathrm{~T} 7
$$

Donde:

TTotal $=$ Tiempo total de la cadena logística despacho diferido.

$\mathrm{T} 4=$ Tramo 4 del despacho diferido.

T7 = Tramo 7 del despacho diferido.

Esta ecuación nos indica que mientras se tiene un tiempo constante de 793,945 horas el despacho diferido variará teniendo en cuenta el comportamiento de los tramos 4 y 7; es decir, mientras mayor sean estos valores, mayor será el tiempo total de despacho diferido.

\section{DISCUSIÓN}

El tiempo promedio de la cadena logística de importación bajo la modalidad de despacho anticipado durante el periodo del 2015 al 2018 fue de 55,2 días, mientras que bajo la modalidad del despacho diferido fue de 41,8 días, según la información analizada y bajo los tramos propuestos para cada modalidad. La SUNAT cuenta con un indicador vinculado al régimen de importación para

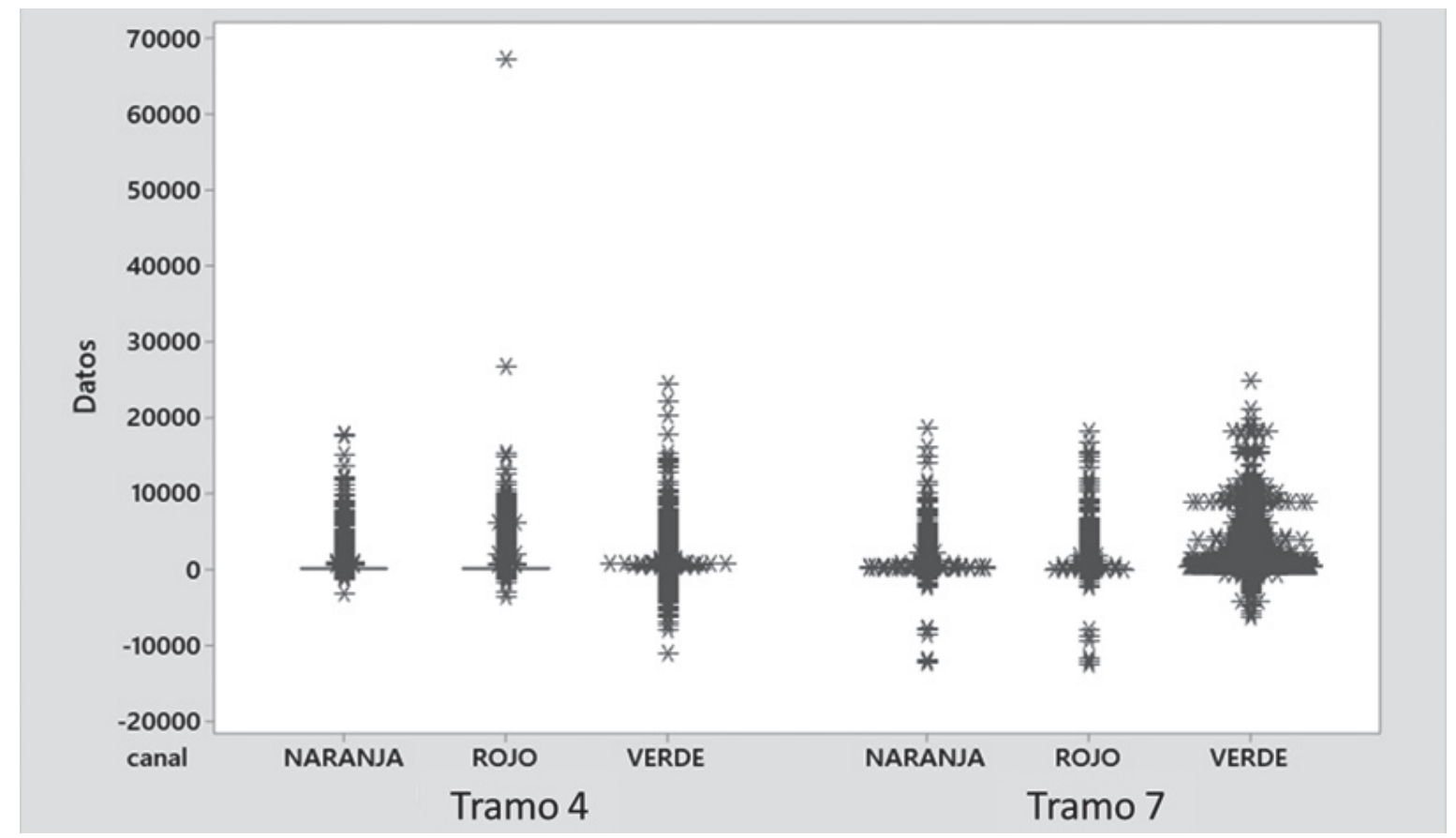

Figura 3. Gráfico de dispersión de DAM de los tramos 4 y 7 respecto a la mediana. Los asteriscos representan a las DAM y su comportamiento por canal de control ( $\mathrm{V}=$ verde, $\mathrm{D}=$ naranja y $\mathrm{F}=$ rojo) dentro de los tramos analizados. Elaborado en base a la información proporcionada por la SUNAT. 


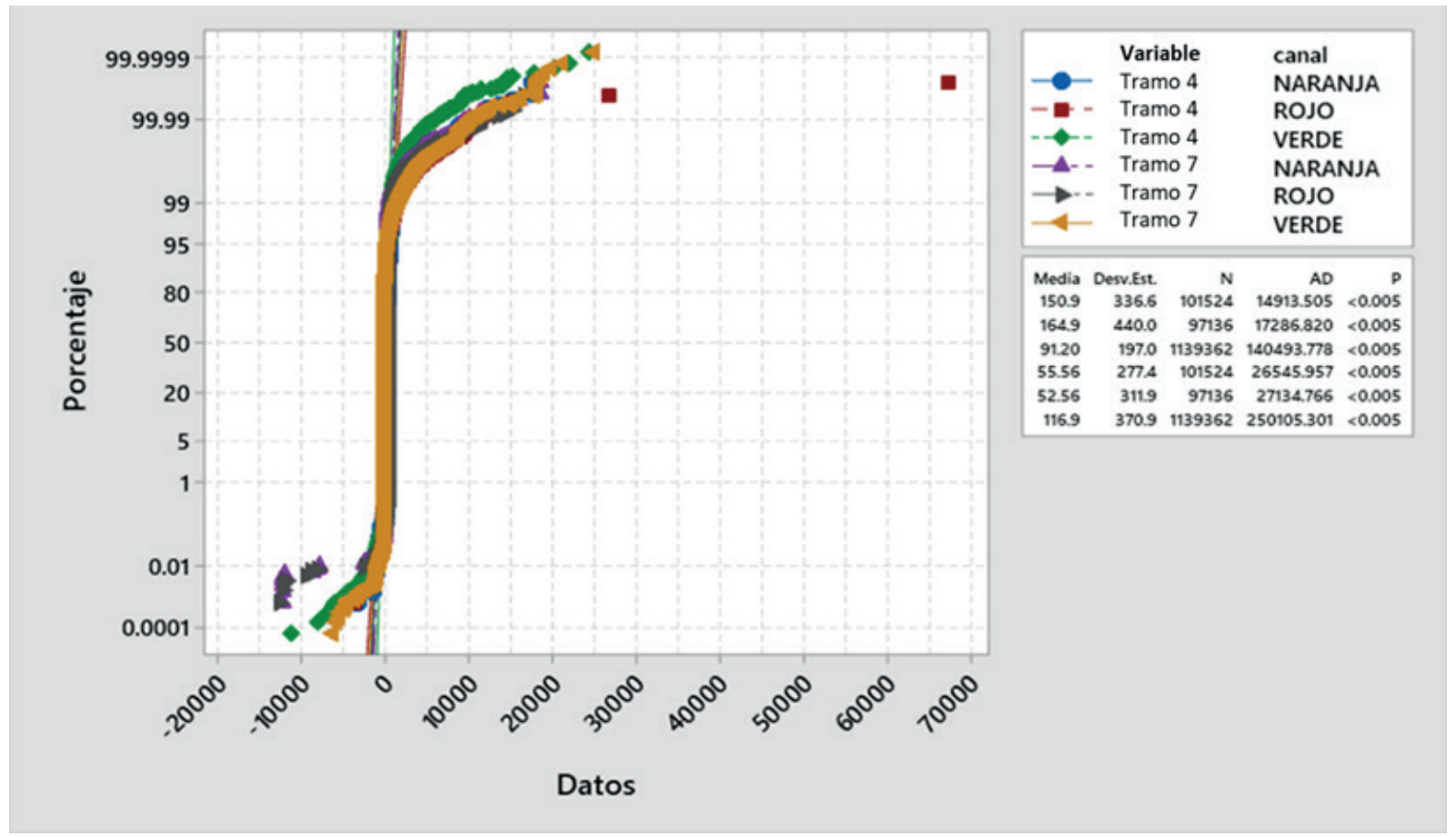

Figura 4. Prueba de normalidad tramos 4 y 7 (Normal - 95\% de IC). Elaborado en base a la información proporcionada por la SUNAT.

el consumo llamado Tiempo Total de Liberación de Mercancías (TTLM), este mide el tiempo total de despacho desde la llegada del medio de transporte hasta la autorización de levante de las mercancías consignadas en la DAM. Como es evidente, la SUNAT no realiza una medición de toda la cadena logística, dentro del despacho diferido se aprecia el establecimiento de tramos para su medición, mas no en el despacho anticipado. Si se hace un comparativo de lo indicado por SUNAT con lo analizado en presente estudio, se colige que el despacho diferido vendría a ser el óptimo para el importador; por tanto, si la SUNAT pretende eliminar en el futuro el despacho diferido y mantener solo el despacho anticipado, deberá de mejorar los tiempos de atención y realizar un estudio de todos los tramos que componen esta cadena logística en una labor conjunta con otros operadores del comercio internacional (transportistas, depósitos temporales, terminales portuarios, etc.) a fin de mejorar toda la cadena logística y no únicamente la parte de interacción entre el importador y la SUNAT.

\section{REFERENCIAS}

Agencia de los Estados Unidos para el Desarrollo Internacional - USAID (2012). Guía de orientación para el usuario del despacho anticipado. Perú: Tarea Asociación Gráfica Educativa.
Agencia Peruana de Noticias - ANDINA (2018). SUNAT lanza primera herramienta virtual para reducir tiempo de importaciones. Recuperado de: https://andina. pe/agencia/noticia-sunat-lanza-primera-herramienta-virtual-para-reducir-tiempo-importaciones-718670.aspx.

Asociación Peruana de Agentes Marítimos - APAM (2018). SUNAT: Con el despacho anticipado, podemos multiplicar las operaciones de comercio. Recuperado de: https://www.apam-peru.com/web/sunat-con-el-despacho-anticipado-podemos-multiplicar-las-operaciones-de-comercio/.

Cosio, F. (2017). Curso de Derecho Aduanero. Perú: Jurista Editores.

Doing Business (2019). Capacitación para reformar: Recuperado de: https://espanol.doingbusiness.org/es/ reports/global-reports/doing-business-2019

El Peruano. (2008). Decreto Legislativo $N^{\circ} 1053$ - Ley General de Aduanas. Recuperado de: https://diariooficial. elperuano.pe/Normas

El Peruano. (2018). Decreto Legislativo $N^{\circ} 1433$ - Decreto Legislativo que modifica la Ley General de Aduanas. Recuperado de: https://diariooficial.elperuano.pe/Normas 
Estrada, A. (2018). Análisis de la conveniencia de la obligatoriedad del despacho anticipado de importación en el marco de D.L. 1235 que modifica articulo 131 Ley General de Aduanas (tesis de pregrado). Universidad de Lima, Lima, Perú.

Guadalupe, J. (2019). SUNAT: Despacho anticipado y cultura de la imposición. Gestión. Recuperado de: https:/gestion.pe/blog/zonaintercambio/2019/09/ despacho-anticipado-y-cultura-de-la-imposicion.ht$\mathrm{ml} /$ ?ref=gesr.

Perú, Superintendencia Nacional Aduanas y de Admninistración Tributaria. SUNAT. (2018). Memoria Anual. Recuperado de: http://www.sunat.gob.pe/cuentassunat/planestrategico/memoria/memoria2018.pdf.
PQS Perú. (2018). SUNAT: despacho anticipado hará que importadores liberen mercancías en menos tiempo. Recuperado de: https://www.pqs.pe/economia/sunat-despacho-anticipado-hara-que-importadores-liberen-mercancias-en-menos-tiempo

Romero, M. (2016) Pruebas de bondad de ajuste a una distribución normal. Revista Enfermería del Trabajo,6(3), pp.105-114 . Recuperado de: file://C:/Users/ Lenovo/Downloads/Dialnet-PruebasDeBondadDeAjusteAUnaDistribucionNormal-5633043.pdf 
